\title{
Correction: Aortic valve replacement for Libman-Sacks
} endocarditis

Keenan JB, Janardhanan R, Larsen BT, et al. Aortic valve replacement for Libman-Sacks endocarditis. BMJ Case Reports 2016; doi:10.1136/bcr-2016-215914

The revised author list of this paper is as follows:

Jack B Keenan, Taufiek Konrad Rajab, Rajesh Janardhanan, Brandon T Larsen, Zain Khalpey BMJ Case Rep 2016. doi:10.1136/bcr-2016-215914corr1 